\title{
Commentary \\ Ablation of atrial fibrillation: a procedure come of age?
}

J Marcus Wharton

Section of Clinical Cardiac Electrophysiology, Duke University Medical Center, Durham, North Carolina, USA

Correspondence: J Marcus Wharton, MD, Box 3816, Duke University Medical Center, Durham, NC 27710, USA. Tel: +1919 681 6478;

fax: +19196819260; e-mail: whart002@mc.duke.edu

Received: 6 March 2001

Accepted: 13 March 2001

Published: 5 April 2001
Curr Control Trials Cardiovasc Med 2001, 2:67-70

(C) 2001 BioMed Central Ltd

(Print ISSN 1468-6708; Online 1468-6694)

\begin{abstract}
Various approaches for catheter ablation of focal initiators of atrial fibrillation or the substrate for maintaining atrial fibrillation have evolved over the past 5 years. Despite these advances, there are still a large number of unresolved issues regarding the efficacy and safety of these procedures as well as optimal patient selection for the different approaches. These uncertainties raise questions about the applicability at the present time of atrial fibrillation as front-line therapy within the community.
\end{abstract}

Keywords: ablation, atrial fibrillation, pulmonary veins

During the past 5 years, great strides have been made in unraveling the complex interplay of factors that are responsible for initiating and maintaining atrial fibrillation (AF). According to our present understanding of $A F$ (which is still in its infancy), AF is initiated by a number of possible supraventricular tachyarrhythmias, most commonly premature atrial contractions or rapid, often nonsustained, atrial tachycardias that arise from the pulmonary veins and, less commonly, nonpulmonary vein sites [1-3]. These are generally 'focal' arrhythmias; that is, they can be mapped to relatively small areas of less than a few millimeters. These focal triggering arrhythmias may initiate true AF, which is due to a complex series of multiple wavefronts that simultaneously activate both the right and left atria in a nonrepeating pattern or in minimally repeating patterns [4].

The ability to maintain AF is dependent on a number of factors, such as atrial size, mass, stretch and fibrosis, heterogeneity of activation and repolarization, continued activation of triggering arrhythmias to prevent organization and termination, and autonomic tone $[5,6]$. If the appropriate substrate is not present, AF once initiated will not be maintained and will spontaneously terminate. Thus, the interac- tion between initiators and substrate determine whether the clinical presentation is that of paroxysmal or persistent AF. In addition, a host of factors serve to modulate both the initiators and substrate of $A F$, including autonomic tone, volume status, body position, drugs, and electrical and structural remodeling that occur as a direct consequence of AF [5-7]. Thus, AF can be considered as a complex interplay of initiators, substrate, and modulating factors.

The goal of AF ablation approaches may be either palliative or curative. Palliative procedures are aimed at nonpharmacological rate control by creation of complete heart block (AV node ablation with permanent pacemaker placement) or high-grade AV block (AV nodal modification without pacemaker). These approaches obviously do not prevent $\mathrm{AF}$ from continuing or recurring, not do they eliminate the need for antiarrhythmic drugs or anticoagulation. More recently, approaches have been developed to prevent recurrent AF. These curative ablation approaches for AF are aimed at either eliminating or isolating AF initiators, and/or alterating of atrial substrate in order to prevent the maintenance of AF. Either approach should prevent recurrence of sustained AF. What limits all of these attempts at 
AF ablation is that we do not understand the full range of events that initiate and/or maintain AF. Nonetheless, progress has been made toward successful ablation of AF in a number of clinical conditions, such as AF in the setting of no or minimal heart disease. These attempts have significantly contributed to our present understanding of the pathogenesis of AF. The question presented by this rapid growth in our knowledge is whether AF ablation is sufficiently mature to serve as frontline therapy for $\mathrm{AF}$, with widespread application within the community.

\section{Ablation of focal initiators of AF}

The greatest advances have been made in the ablation of the focal atrial arrhythmias that are responsible for initiating and, in some cases, maintaining AF. Although regular rapid atrial tachyarrhythmias were shown in animal models to be possible causes of $\mathrm{AF}$ as long as 50 years ago [8] and were observed to be the initiating events of $A F$ in humans 30 years ago [9], the precise nature of these initiators in humans remained poorly understood until recently. Haissaguerre and coworkers $[1,3]$ and others [2] recently showed that atrial tachycardias, which arise most typically within the residual left atrial musculature that surrounds the pulmonary veins as they approach the left atrium, are responsible for initiating most $\mathrm{AF}$, at least in those patients with no or minimal heart disease [1-3]. Ablation of these atrial tachycardias has been shown to cure $A F$ in patients with frequent atrial ectopy and insignificant cardiac disease. A number of retrospective and uncontrolled studies [1-3] have shown that focal AF ablation may be curative in $60-85 \%$ of these patients. Variables that are related to success rates include patient inclusion criteria, ablation approaches, severity of underlying atrial disease, number of initiator sites, and the duration of AF. Although none of these studies had parallel control groups, most of the patients had high frequencies of $\mathrm{AF}$ before ablation, and the lack of symptomatic episodes after successful ablation strongly suggests that the procedure is effective.

\section{Limitations of focal AF ablation}

Despite the encouraging initial reports, ablation of focal initiators of AF is still in its infancy. Several issues need to be addressed. To date, only heavily selected groups of patients have been studied. Typically, these groups have included younger patients with no or minimal heart disease, and with paroxysmal AF, high-density atrial ectopy, and a priori assessment that they had 'focal AF'. The efficacy of these procedures applied to unselected patient populations with paroxysmal AF is not known, and only limited data are available regarding efficacy for patients with persistent and chronic AF. Preliminary studies that evaluated patients with persistent AF [3] have demonstrated more modest results, and preliminary results for patients with chronic AF are very disappointing. Furthermore, there is little available data regarding the efficacy of the procedure in the setting of significant cardiac disease. More information regarding optimal patient selection for focal AF ablation approaches is needed.

In reported studies regarding the ablation of AF initiators, follow up is limited to several months, or at best a couple of years. Insensitive follow-up techniques may result in considerable errors of omission, and may overestimate longterm cure rates. In addition, conversion of symptomatic to asymptomatic AF could occur as a consequence of these procedures and not be detected on simple symptom reviews during follow up. Appropriate means for long-term follow up in these trials needs to be determined. However, the limitations of random 24-h Holter recordings once every month or a few seconds of event recordings every week are obvious in a disease process that may manifest itself only for a few hours every other month. Recently developed arrhythmia-triggered (as well as patient-activated) event monitors may be useful in this situation for more precise determination of whether the end result is truly curative or just quantitative suppressive of AF events.

Given the relatively short duration of available follow up for focal AF ablation approaches, we do not know whether these procedures will continue to protect patients over years and decades. Importantly, we do not understand either the pathogenesis or the natural history of these initiating arrhythmias. Whether additional initiators will evolve over the years is not known, and will remain unknown until long-term follow-up study results are available. In addition, although most of the initiating arrhythmias appear to arise within the pulmonary veins, the applicability of this assumption to larger, more varied groups of patients with $\mathrm{AF}$ remains to be tested. Thus, the efficacy of empiric pulmonary vein isolation procedures in the diversity of patients with $A F$ is unknown.

Of major concern with pulmonary vein isolation or ablation procedures is the occurrence of partial or complete stenosis of the pulmonary veins [10]. Symptomatic stenosis is detected in fewer than $5 \%$ of patients, and is due to complete or near-complete stenosis of one or more pulmonary vein [1-3]. However, up to $42 \%$ of patients may have some degree of pulmonary vein stenosis [2]. Although tolerance of these mild degrees of stenosis is excellent, the long-term sequelae are not known. Cases of pulmonary hypertension as a consequence of pulmonary vein ablation and stenosis have been reported [11]. Although stenting of the stenosis may be effective acutely, the long-term patency of these stents is unknown.

\section{Catheter-based maze procedures}

Even less is known about procedures that are aimed at eliminating the ability of the atria to maintain AF. Curative ablation of AF can be achieved during open-heart surgery by surgically isolating the pulmonary veins from the atria, 
dividing the right and left atria into several connected, dead-end corridors in continuity with the sinus node, and excising the atrial appendages [12]. The surgically created lines of conduction block created by the connected deadend corridors prevent the wandering wavelets of re-entry during AF from sustaining. The surgical maze procedures have been shown to be effective treatments for paroxysmal, persistent, and chronic AF. Although open-chest procedures are required, the cure rate is approximately $85 \%$, with substantial morbidity and some mortality attendant to the surgical procedures [12]. Following these principles, multiple investigators have shown [13-16] that creation of multiple long lines of conduction block in both the right and left atria to simulate in part the surgical maze procedure may cure some AF patients. Although the initial retrospective analyses looked exceptionally promising, with up to $87 \%$ cure rates [16], two ongoing prospective studies $[17,18]$ of biatrial lesion sets have been less optimistic, with success rates of approximately $50 \%$ or less. Although a greater number of patients have less frequent $\mathrm{AF}$ on antiarrhythmic drugs, this end-point is subjective, is difficult to quantify, and is subject to bias. More importantly, rates of major complications in the prospective studies have been unacceptably high, occurring in approximately one-quarter of patients. Although symptomatic control with continued antiarrhythmic drug therapy can be achieved in the majority of patients, the high rates of serious complications significantly limit application of these procedures to only a few, heavily symptomatic patients, and hamper enrollment of patients in studies evaluating new technologies that are aimed at simplifying the procedure. Limitation of the linear lesions to the right atrium greatly simplifies the procedure and avoids the potential risks associated with creation of linear lesions in the left atrium. Right-atrium-only linear lesion sets rarely cure patients with paroxysmal AF, however, and are ineffective in chronic AF $[16,19,20]$. Selected patients with paroxysmal AF may have high rates of cure [20], but the criteria for selection of these patients are not well defined.

\section{Prophylactic anticoagulation post-ablation}

By eliminating AF with ablation of either the initiators or substrate, it is assumed that the long-term need for chronic anticoagulation for stroke prophylaxis is avoided. Although uncontrolled data from patients after surgical maze procedures appears to support this contention [21], there are no long-term data regarding AF ablation procedures. Anecdotal cases of late postablation cerebroembolic events have been reported. These may be due to emboli from a number of sources, including the following: pre-existing left atrial thrombi; lesion-related thrombus in the left atrium or pulmonary veins; thrombus from diminished left atrial transport; paradoxic emboli across persistence flow communications from trans-septal access sites; continued undetected atrial fibrillation; and valvular or peripheral vascular disease. Thus, successful ablation does not necessarily exclude the need for chronic anticoagulation. Further data from the individual procedures are needed in order to determine the advisability of withdrawal of anticoagulation after AF ablation.

\section{What needs to be done}

As with all issues regarding nonpharmacologic therapy, prospective, controlled trials comparing various ablation approaches with traditional antiarrhythmic drug therapy or atrioventricular nodal ablation and pacemaker placement need to be performed. These trials can address such issues as comparative efficacy, complication rates, quality of life indices, and cost-effectiveness. Because mortality rates for conventional and new ablation approaches are very low, it is unlikely that trials of sufficient power will ever be performed to determine whether mortality benefits exist. In addition, there are several approaches for treatment of initiators. These include ablation directed at spontaneously discharging foci, isolation of pulmonary veins with spontaneously occurring arrhythmias, and empiric pulmonary vein isolation of all pulmonary veins with a muscular sleeve, regardless of whether an arrhythmogenic focus is identified. Likewise, there are different approaches for performing linear lesions for substrate modification. Various technologies have been or are being developed to ablate initiators and modify substrate. As these approaches are developed, prospective comparisons are needed to determine which approaches and technologies have the greatest efficacy and safety.

It is clear from the available information that curative ablation approaches for AF are rapidly advancing, but too little is known at present to advocate widespread implementation. In particular, given the potential for serious complications, even with ablation of AF initiators in healthy individuals, it is difficult to advocate ablation as frontline therapy in advance of traditional pharmacologic approaches. Furthermore, the procedures are technically difficult, arduous, time-consuming, and are not cost-effective. Given the risks of serious complications, even in experienced hands, a loud note of caution must be expressed concerning widespread application by individuals who are inexperienced in technical aspects of the procedure, such as trans-septal catheterization, pulmonary vein ablation, and linear lesion creation in the left atrium. Appropriate educational guidelines need to be established in order to ensure safe application of these approaches in the community.

\section{References}

1. Haissaguerre $M$, Jais $P$, Shah DC, Garrigue $S$, Takahashi A, Lavergne T, Hocini M, Peng JT, Roudant R, Clementy J: Electrophysiological end point for catheter ablation of atrial fibrillation initiated from multiple pulmonary vein foci. Circulation 2000, 101:1409-1417.

2. Chen SA, Hsieh MH, Tai CT, Tsai CF, Prakash VS, Yu WC, Hsu TL, Ding YA, Chang MS: Initiation of atrial fibrillation by ectopic beats originating from the pulmonary veins: electrophysiological characteristics, pharmacological responses, and effects of radiofrequency ablation. Circulation 1999, 100:1879-1186. 
3. Haissaguerre $M$, Jais $P$, Shah DC, Arentz T, Kalusche D, Takahashi A, Garrigue S, Hocini M, Peng JT, Clementy J: Catheter ablation of chronic atrial fibrillation targeting the reinitiating triggers. J Cardiovasc Electrophysiol 2000, 11:2-10.

4. Allessie MA, Lammers WJEP, Bonke FIM, Holen J: Experimental evaluation of Moe's multiple wavelet hypothesis of atrial fibrillation. In: Cardiac Arrhythmias. Edited by Zipes DP, Jalife J. New York: Grune and Stratton; 1985:265-276.

5. Ramanna H, Hauer RN, Wittkampf FH, de Bakker JM, Wever EF, Elvan A, Robles De Medina EO: Identification of the substrate of atrial vulnerability in patients with idiopathic atrial fibrillation. Circulation 2000, 101:995-1001.

6. Huang JL, Wen ZC, Lee WL, Chang MS, Chen SA: Changes of autonomic tone before the onset of paroxysmal atrial fibrillation. Int J Cardiol 1998, 66:275-283.

7. Wijffels MC, Kirchof CJ, Dorland R, Allessie MA: Atrial fibrillation begets atrial fibrillation. A study in awake chronically instrumented goats. Circulation 1995, 92:1954-1968.

8. Scherf D: Studies on auricular tachycardia caused by aconitine administration. Proc Soc Exp Biol Med 1947, 64:233-239.

9. Bennett MA, Pentecost BL: The pattern of onset and spontaneous cessation of atrial fibrillation in man. Circulation 1970, 41:981-988.

10. Taylor GW, Kay GN, Zheng X, Bishop S, Ideker RE: Pathological effects of extensive radiofrequency energy applications in the pulmonary veins of dogs. Circulation 2000, 101:1736-1742.

11. Robbins IM, Colvin EV, Doyle TP, Kemp WE, Loyd JE, MeMahon WS, Kay GN: Pulmonary vein stenosis after catheter ablation of atrial fibrillation. Circulation 1998, 98:1769-1775.

12. Sundt TM, Camillo CJ, Cox JL: The maze procedure for cure of atrial fibrillation. Cardiol Clin 1997, 15:739-748.

13. Maloney JD, Milner L, Barold S, Czerska B, Markel M: Twostaged biatrial linear and focal ablation to restore sinus rhythm in patients with refractory chronic atrial fibrillation: procedure experiences and follow-up beyond 1 year. Pacing Clin Electrophysiol 1998, 21:227-2532.

14. Ernst S, Schluter M, Ouyang F, Khanedani A, Cappato R, Hebe J, Volkmer M, Antz M, Kuck KH: Modification of the substrate for maintenance of idiopathic human atrial fibrillation: efficacy of radiofrequency ablation using nonfluoroscopic catheter guidance. Circulation 1999, 100:2085-2092.

15. Jais $P$, Shah DC, Haissaguerre $M$, Takahashi $A$, Lavergne $T$, Hocini M, Garrigue S, Barold SS, Le Metayer P, Clementy J: Efficacy and safety of septal and left-atrial linear ablation for atrial fibrillation. Am J Cardiol 1999; 84:139R-146R.

16. Cannom DS: Atrial fibrillation: nonpharmacologic approaches: an evolving era. Am J Cardio/ 2000, 85:25D-35D.

17. Kay GN, Ellenbogen KA, Wood MA, Calkins H, Walcott GP, Hall $\mathrm{JA}$ : Linear phased radiofrequency catheter ablation in the left and right atria for the treatment of atrial fibrillation. Circulation 1999, 100:I-779.

18. Haines DE, Hummel JD, Kalbfleisch SJ, Daoud EG, Avital B, Langberg JJ, Leon AR, Epstein LM: Bi-atrial linear ablation with the multiple electrode catheter ablation (MECA) system in patients with atrial fibrillation [abstract]. PACE 2000, 23:567.

19. Haissaguerre M, Jais P, Shah DC, Gencel L, Pradeau V, Garrigues S, Chouairi S, Hocini M, Le Metayer P, Roudant R, Clementy J: Right and left atrial radiofrequency catheter therapy of paroxysmal atrial fibrillation. J Cardiovasc Electrophysiol 1996, 7:1132-1144.

20. Gaita F, Riccardi R, Calo L, Scaglione M, Garberoglio L, Antolini R, Kirchner M, Lamberti F, Richiardi E: Atrial mapping and radiofrequency catheter ablation in patients with idiopathic atrial fibrillation. Electrophysiological findings and ablation results. Circulation 1998, 97:2136-2145.

21. Ad N, Cox JL: Stroke prevention as an indication for the Maze procedure in the treatment of atrial fibrillation. Semin Thorac Cardiovasc Surg 2000, 12:56-62. 